\title{
Properties of Quantum Beams and Their Applications
}

\author{
Naoaki Fukuda ${ }^{1,2}$, Toshio Takiya ${ }^{1} \&{\text { Min } \operatorname{Han}^{3}}^{3}$ \\ ${ }^{1}$ Hitachi Zosen Corporation, Osaka, Japan \\ ${ }^{2}$ Research Center for the 21st Century, Osaka Prefecture University, Osaka, Japan \\ ${ }^{3}$ Department of Materials Science \& Engineering, Nanjing University, Nanjing, China \\ Correspondence: Toshio Takiya, Hitachi Zosen Corporation, Osaka, Japan. E-mail: takiya@hitachizosen.co.jp
}

Received: January 21, 2018

Accepted: February 28, 2018

Online Published: March 21, 2018

doi:10.5539/apr.v10n2p30

URL: https://doi.org/10.5539/apr.v10n2p30

\begin{abstract}
A conceptual formulation of quantum beams and their basic properties are presented. The present status and outlook of their industrial applications are also discussed. Quantum beams are highly directional energy beams consisting of quantum-mechanical particles characterized by wave-particle duality. They are a concept developed out of need in industry, and, together with quantum mechanics developed during the turn of the century, have been applied to semiconductor and medical industries. The quantum beams can be classified by penetrating or ionizing power. X-rays and neutron beams are classified into those with high penetrating power, and the beams of alpha particles are classified into those with high ionizing power. Electron beams fall in between, giving rise to their unique intermediate property. Their chemical and biological effects are used in modifying the properties of materials or sterilizing food and beverage containers. Finally, we discuss the importance of developing further advanced accelerator technologies which can produce high-energy quantum beams, which will be necessary to chart our future research in yet unknown areas of science. In doing so, profit should not be the only goal; contribution to a sustainable society should be considered as well.
\end{abstract}

Keywords: quantum beams, quantum mechanics, electron beams, penetrating power, ionizing power, beam acceleration technology, trans-science

\section{Introduction}

Quantum beams are a concept that includes conventional electromagnetic radiation, such as X- and gamma rays; particle radiation, such as ion beams and heavy particle beams (Liss, 2017); and various types of laser beams.

Electricity generation in matter irradiated by incident light was discovered in 1839 . This discovery prompted research and development in the field related to the interaction between light and matter. In this field, research and development have been focused on theories explaining how the interaction induces changes in matter or affects the behavior of electromagnetic waves, elucidating various phenomena, and the results have been applied to industry. Quantum mechanics was thus firmly established in the 20th century, and the advancement of computational technologies enabled the practical application of quantum mechanics. In the second half of the 20th century, semiconductor industry, one of the areas of industrial applications of quantum mechanics, flourished, and nuclear power generation based on nuclear fission technology was realized. New research fields aimed at developing an environmentally-friendly energy-saving society using quantum mechanics, such as superconducting materials and single-electron devices, emerged. This evolution continues; in the 21st century, new scientific technologies and industries based on quantum mechanics are about to unfold in yet unknown areas. In such a time like this, what should we do? We should look back at our past first; we must reevaluate foundational technologies developed in the 20th century. In that context, quantum beams can be one of the new foundational technologies (Fukuda, 2017).

A recently observed trend is a unified approach to various energy beams, such as electromagnetic radiation, particle radiation, and laser, which used to be treated separately, for a broader application in industry. For instance, penetrating power of electromagnetic radiation has been applied to medical examination devices, such as radiography, whereas ionizing power of particle radiation has been applied to medical treatment devices used in cancer therapy, etc. We consider these energy beams a foundational technology that is instrumental to develop new industries, in addition to the traditional areas of application; in other words, they are a broadly encompassing and unified technology that can be applied to, for example, precision measurements and micro-processing as part 
of device technology, molecular cross-linking technology as their application to chemistry, biocidal and sterilization technology as their application to biology (Furuta et al., 2002; Furuta \& Fukuda, 2016). This new unified technology is referred to as quantum beam here.

In this article, we will give an outline of the basic properties of quantum beams that users should be aware of and their application examples. We will further discuss some noteworthy points in applying advanced quantum beam technologies to industrial processes as well as the future of quantum beams.

\section{Basic Properties of Quantum Beams}

\subsection{Definition of Quantum Beam}

First, let us explain the terminology of quantum beam, which is not quite well known to the public.

The word "quantum" refers to an existence or state that has both particle-like and wave-like properties. We must introduce this concept when we consider the motion of any microscopic systems involving electrons or photons. In macroscopic systems, forces experienced by an "object" are analyzed by classical mechanics to predict what kinds of "phenomena" will occur, whereas in microscopic systems, there is no clear distinction between an "object" and "phenomena". In other words, these two concepts were mediated by quantum mechanical particles. A quantum mechanical particle, such as an electron or a photon, is referred to as quantum here. The quantum existence or state is described by a function of a complex variable called wave function. One of the objectives of quantum mechanics is to determine this function and use it to predict the quantum existence or state. The uncertainty principle, which is the key fundamental principle of quantum mechanics, shows that we cannot observe both particle-like and wavelike properties of particle simultaneously, or put it differently, we cannot measure the position and momentum of particle simultaneously. This property of quantum mechanical particle is called complementarity, which has been a fundamental concept that defies common sense in mechanics of macroscopic systems.

Quantum beams may be defined as a collective noun for high-energy directional beams of above-mentioned quanta, such as photons, electrons, neutrons, and heavy ions. Examples of quantum beam include laser, electron beam, neutron beam, ion beam and so forth. The word was coined in Japan to express new guiding concepts of science and technology. It was first used in the World Year of Physics forum held in Tokyo in 2005. It is used worldwide today.

In this section, we will briefly describe quantum mechanics to help you understand unique properties of quantum beams, and then explain their characteristic properties and applications.

\subsection{Birth of Quantum Concepts and Formulation of Theories}

Quantum mechanics was spawned from research and development endeavor to accomplish a stable production of high-quality products. We will give an overview of history of such development here (Kragh, 1999). We hope this story will strike a chord of readers.

The Industrial Revolution, which began toward the end of the 18th century, had brought about a rapid growth of steel industry. Steel, a major product in those days, was made in industrial furnaces. To accomplish an improvement in quality as well as more stable production of steel, researchers and engineers in those days tried to control the temperature inside furnaces, but there was no available means to measure the temperature inside back then. So, they estimated the temperature by observing the light emitted from molten steel. This technique was later applied to other materials as well, which led to a boom of analytical research in the light emitted from heated materials, which further led to research in spectral analysis of intensity and wavelength of light (frequency spectral distribution). By the end of the 19th century, the biggest challenge for physicists back then was to provide a theoretical explanation for empirical findings of the spectral distribution of light emitted from materials.

Max Planck published the quantum hypothesis about radiant energy in 1900. His hypothesis was expressed by the equation

$$
\varepsilon_{n}=n h f
$$

where $\varepsilon_{n}$ is energy of a harmonic oscillator, $n$ is an integer $(n=1,2,3 \ldots), h$ is the Planck constant, and $f$ is the frequency of oscillator. This mathematical expression marked the dawn of quantum mechanics. Classical physics, which thus far dictated the laws of nature, considered changes in any physical quantities continuous, whereas Planck's equation suggested that those changes are always an integer multiple of an elementary unit, i.e. they always take discontinuous or discrete values. This notion was completely new to physics. Planck coined a word "action quantum" for the notion.

Einstein extended Planck's idea further, and published the hypothesis of light quanta (referred to as photons today) in 1905, where light was considered particles (photons) and the energy was described by the equation 


$$
\varepsilon=h f
$$

where $\varepsilon$ and $f$ are energy and frequency of photon, respectively. This hypothesis successfully explained some phenomena that classical physics thus far had failed to explain, such as photoelectric effect, where electrons are emitted from a metal surface illuminated by incident light.

$$
E=m c^{2}
$$

the equation in Einstein's general theory of relativity published in 1907, also led to the development of quantum mechanics. This equation is such a famous one that further explanation may not be necessary, but for sake of clarity, $m$ is mass of a matter and $c$ is the speed of light in this equation.

De Broglie, influenced by Einstein's hypothesis of light quanta, published in 1923 that the notion of wave-particle duality applies not only to light (photons) but also to electrons. Let us derive the de Broglie's general formula here.

The momentum equation for photon can be described as bellow:

$$
p=\frac{\varepsilon}{c}
$$

and wavelength of photon

$$
\lambda=\frac{c}{f}
$$

When equations (2) and (5) are plugged into Equation (4), we get the following de Broglie's equation

$$
\lambda=\frac{h}{p}
$$

De Broglie proposed a bold hypothesis that any matter waves, including electron wave, may be expressed with Equation (6) by using the expression of the momentum $p=m v$, where $v$ is the velocity of matter wave. This hypothesis suggested that matter wave also has the wave-particle duality, which is related by the Planck constant. These hypotheses in quantum physics were verified one by one by various experiments of light and electrons. Subsequently, matrix mechanics by Heisenberg, wave mechanics by Schrödinger, and quantum algebra by Dirac were published. The theories of key components of quantum mechanics were established in 1926.

\subsection{Dawn of Quantum Beam Age and Foundation of Quantum Beam Control}

The first quantum beam used in industry in history was X-ray. The first paper written on X-ray was published in 1895 by Röntgen. In the following year 1896, Becquerel discovered radiation emitted from uranium compounds. This radiation has some characteristic properties: It can pass through matter, and cause fluorescence, ionization, and chemical reactions in photographic plates. These unique properties were first applied to science and medicine, and later to engineering. The greater the area of application, the greater a desire for manipulation of quantum beams. It has been more than 100 years since quantum beam first used in industry; and yet, there still remains a strong desire to manipulate beams. Currently, a number of research and development projects are going on in this area.

We will continue the topic of how to control quantum beams. Electron beams are relatively easier to control than other quantum beams and therefore have been used in industry from the early days. Here we will focus on the electron beams and explain the methods of controlling them. Examining the behavior of electron beams, which have many everyday-life application examples based on their particle and wave properties, will deepen your understanding in quantum beam control.

First, we will explain how to generate electron beams. Perhaps many of the readers know that metals have a special type of electrons, called free electrons. When metals are heated, electrons inside them start to vibrate. The more the metals are heated, the greater the electrons' energy become; eventually, free electrons will be knocked out from the metal surface. Since electrons are negatively charged, applying negative electric potential to metal creates an environment that facilitates the emission of electrons from the metal surface. Placement of a positively charged electrode in the direction of electron emission creates an electric field where negatively charged electrons accelerate toward the positive pole (plus terminal). Figure 1 illustrates the mechanism of acceleration of electrons. 


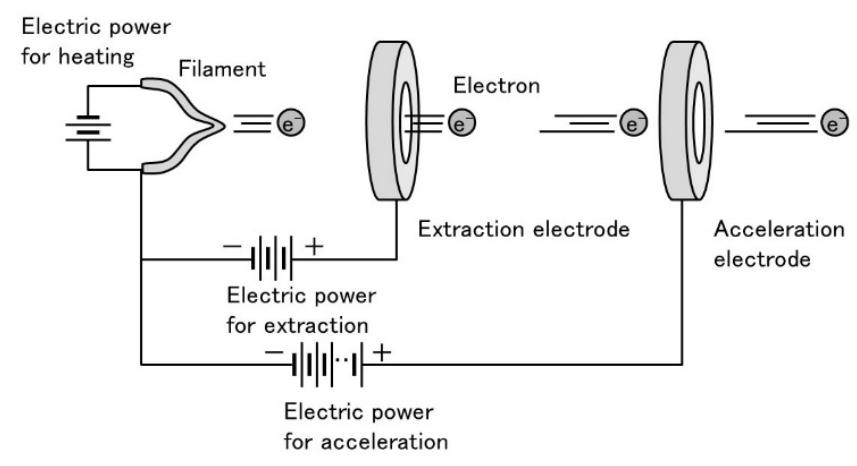

Figure 1. Schematic diagram showing the mechanism of electron beam acceleration

When electrons are accelerated along the electric field, they gain kinetic energy $(\mathrm{eV})$ equivalent to that of an elementary charge (e) multiplied by an electric potential difference between two electrodes, or accelerating voltage (V). This statement is equivalent to the following: Changing accelerating voltage enables us to control electron's kinetic energy.

As charged particles, electrons obey Fleming's left-hand rule in an applied magnetic field, which allows manipulation of the direction of irradiation. Cathode-ray tube televisions (CRT TVs) use this property. Figure 2 presents the mechanism of CRT TVs.

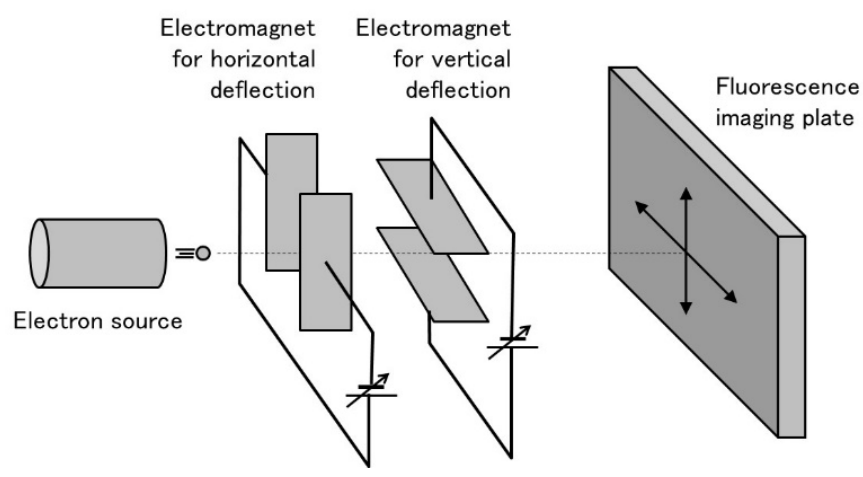

Figure 2. Schematic diagram showing the mechanism of CRT TVs

As shown in Figure 2, fluorescent material is coated on a CRT display, which is scanned with electron beams either in a lateral or longitudinal direction. The beams are bent with the use of bending (deflection) electromagnet. When the beams hit the fluorescent material, the material emits light. CRT TVs employ these phenomena to display images on the screen.

Next, we will explain another characteristic property of electron beams that can be applied to industry. The wave length of electron beam $\lambda$ can be expressed with the following equation:

$$
\lambda=\frac{h}{\sqrt{2 m e V}}
$$

This equation can be derived from the equation of motion of electron beam

$$
e V=\frac{1}{2} m v^{2}
$$

the momentum of matter wave $p=m v$, and de Broglie's equation as shown by Equation (6). Here, $m$ is constant being the mass of electron, and so are $e$ and $h$. Equation (7) tells us that the wavelength of electron beam $\lambda$ is a function of the accelerating voltage $\mathrm{V}$ only. In other word, the greater the accelerating voltage, the greater the kinetic energy of electron and the shorter the wavelength of electron. Figure 3 shows the wavelength-kinetic energy relations of industrial electron beams and other various types of electromagnetic radiation. 


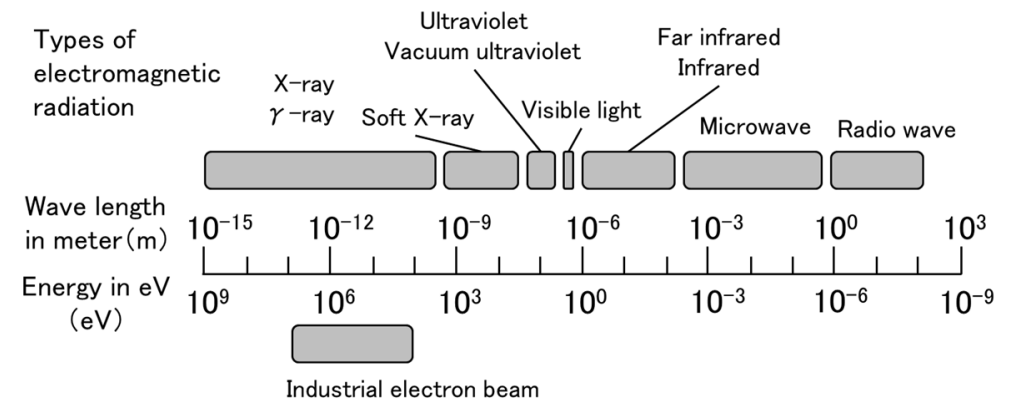

Figure 3. Wavelength and energy of various types of electromagnetic radiation

As shown in Figure 3, industrial electron beams have an extremely short wavelength that is almost four orders of magnitude shorter than visible light. Electron microscopes enables us to see an object at a much smaller scale than optical microscopes. This is made possible by improving microscope resolution by reducing the wavelength of electron beam, which in turn is made possible by increasing the accelerating voltage. This is a typical application example based on the characteristic property of electron beam.

\section{Industrial Applications of Quantum Beams}

\subsection{Interaction between Quantum Beams and Matter}

Interaction between quantum beams and matter depends on the types of irradiated quantum beam - especially, by the charge and mass of particles that constitute the beam (Yu et al., 2003; Hoseinimotlagh \& Zareie, 2014). The underlying science is entanglement of nuclei (positrons and neutrons), electrons, and photons. Examples of the principal interaction effects include ionization, excitation, photoelectric effect, Rayleigh scattering, Compton scattering, positron-electron pair production, bremsstrahlung, and electron-positron annihilation.

Let us change our perspective here. If we look at the macroscopic effects of quantum beams, characteristic properties of various quantum beams can be classified by two properties: penetrating power and ionizing power. Table 1 summarizes the characteristic properties of various quantum beams.

Table 1. Penetrating and ionizing power for various types of electromagnetic radiation and particle beams

\begin{tabular}{|c|c|c|c|c|}
\hline Typ & & $\begin{array}{c}\text { Penetrating } \\
\text { power }\end{array}$ & $\begin{array}{l}\text { Ionizing } \\
\text { power }\end{array}$ & Source \\
\hline \multirow{2}{*}{$\begin{array}{c}\text { Electromagnetic } \\
\text { wave }\end{array}$} & X-ray & strong & weak & $\begin{array}{l}\text { Radioactive } \\
\text { substance } \\
\text { / Accelerator }\end{array}$ \\
\hline & $\gamma$-ray & strong & weak & $\begin{array}{l}\text { Radioactive } \\
\text { substance }\end{array}$ \\
\hline $\begin{array}{c}\text { Natural particle } \\
\text { beam }\end{array}$ & Neutron & strong & weak & $\begin{array}{l}\text { Radioactive } \\
\text { substance } \\
\text { / Accelerator }\end{array}$ \\
\hline \multirow{4}{*}{$\begin{array}{c}\text { Charged particle } \\
\text { beam }\end{array}$} & Electron & medium & medium & Accelerator \\
\hline & Beta particle & medium & medium & $\begin{array}{l}\text { Radioactive } \\
\text { substance }\end{array}$ \\
\hline & Proton & weak & strong & Accelerator \\
\hline & Alpha particle & weak & strong & $\begin{array}{l}\text { Radioactive } \\
\text { substance }\end{array}$ \\
\hline
\end{tabular}

As shown in Table 1, electrically neutral radiation, such as X-ray, has a great penetrating but poor ionizing power. When this type of electromagnetic radiation is directed to a material, it interacts with electrons in the atomic orbitals of atoms that constitute the material, and loses its kinetic energy. The more the number of electron in the atomic orbitals, the more frequently they interact with incident electromagnetic radiation. The frequent interaction impedes the penetration. Hence, the fewer the number of orbital electrons, the deeper the penetration of radiation.

Neutron beams are also electrically neutral. As is the case with electromagnetic radiation, neutron beams have a great penetrating but poor ionizing power in matter. Since neutrons have no charge, they do not cause direct 
ionization, but do cause indirect ionization. How are neutrons different from the electromagnetic radiation? Neutrons primarily interact with atomic nuclei.

On the other hand, charged particles, such as alpha particles and electrons, have a less penetrating but greater ionizing power than electromagnetic radiation. These charged particles experience the effect of electric field in matter, impart part of their energy to orbital electrons or nuclei of atoms, and lose their speed. This distance that the particles travel in matter until they stop is called the range of the particle. The range depends on the mass, energy, etc. of incident particle. Heavy particles, such as alpha particles, characteristically cause the greatest ionization in surrounding atoms towards the end of their travel in matter just before they come to rest. In contrast, electrons, while imparting energy to orbital electrons and nuclei, experience backscattering themselves because of their small mass. The backscattering alters the direction of the incident electrons and slows down their speed. This is called multiple scattering. In this case, the range becomes probabilistic; that is, some incident electrons penetrate deep inside matter and others lose speed in shallows. The range must be defined based on the relation of the thickness of matter and ionizing strength, which is referred to as the effective range.

From here for the remainder of this section, as with Section 2.3, we focus on electron beams and explain phenomena occurring inside matter in more specific details. As shown in Table 1, the properties of electron beams fall between electromagnetic beams, such as X-ray, and "massive" particle beams, such as alpha particles; hence, they are characterized by a moderately great penetrating and ionizing power.

When electron beams are irradiated on a material, negatively charged electrons are decelerated by forces exerted by orbital electrons in atoms or molecules that constitute the material, and impart part of their energy to the orbital electrons and atomic nuclei, giving rise to secondary electrons and X-rays. This process propagates successively, and the incident electrons gradually lose their energy. The secondary electrons in the material cause ionization and excitation of atoms or molecules that constitute the material, giving rise to active species. These active species react with adjacent atoms and molecules and induce various reactions. The series of these reactions occur successively and becomes sources of macroscopic chemical and biological effects.

\subsection{Chemical and Biological Effects of Electron Beams}

In this section, we will further explain industrial applications of the chemical and biological effects of irradiated electron beams described in the previous section, with their relevant history.

The industrial applications of the chemical effects of electron beams began in cross-linking of polyethylene in the 1950s. Cross-linking is a chemical reaction that uses electron beams to create a three-dimensional molecular bond in a polymer compound. The three-dimensional molecular structure improves various properties, such as physical strength and heat resistance. Presently, the cross-linking technique is applied in manufacturing of tires, heatresistant sheets, heat-shrink tubes, etc. Other applications include polymerization between smaller molecules, which is used for curing of paints. The graft polymerization is a chemical reaction that polymerizes large molecules and small molecules. It is one of polymer modification techniques and used in battery separators, etc. The application examples further include an improved switching property in semiconductor devices, which is made possible by introducing lattice defects. The lattice defects are created by irradiating electron beams. In the application examples presented here, electron beams are adopted for two reasons; fast processing speed at room temperature and easier control.

The damaging effect of irradiated electron beams on deoxyribonucleic acid (DNA) molecules in cells is used in applications of the biological effects of electron beams. The irradiation of electron beams on microorganisms imparts energy to molecules that constitute cells, causing some chemical reactions such as ionization. Ionized molecules are very unstable. They readily react with adjacent molecules. There are two types of chemical reactions: direct reaction and indirect reaction. In direct reactions, electron beams disrupt the DNA molecules that carry information essential to maintaining life. In indirect reaction, ionized molecules disrupt the DNA molecules. If damage caused in the DNA molecules by the said two reactions becomes too large to be repaired by cellular repair mechanisms, microorganisms become inactive and die. This biocidal effect of quantum beams including electron beams has been known for a long time, and now widely adopted in the sterilization of various products including medical equipment. Ethylene oxide gas (EOG), which used to be a prevalent biocidal substance in the sterilization of medical equipment, was found toxic. Subsequent restrictions imposed on the use of EOG helped substantially increase the use of electron beams. Low-energy type electron beam generators $(<300 \mathrm{kV}$ accelerating voltage) developed in recent years have started to be introduced in production lines of beverage industry as a means of sterilizing plastic bottles. Figure 4 shows an example of low-energy electron beam irradiation system. 


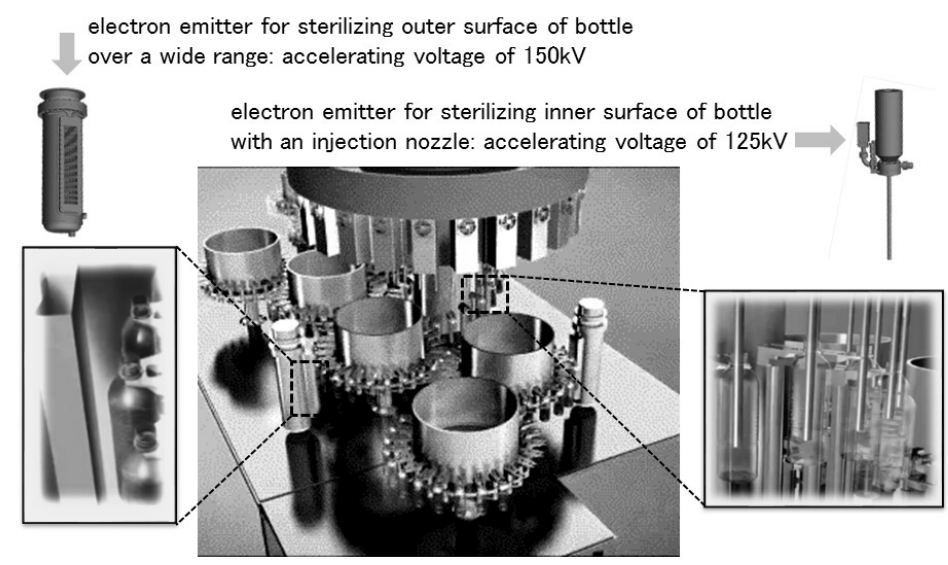

Figure 4. Low-energy electron irradiation system for sterilizing beverage bottles

\section{Future of Quantum Beam}

Accelerator can produce a variety of quantum beams, including electron beams, artificially. The first artificial beam was produced in 1928. Since then, accelerators have been developed primarily to aim at the production of higher energy beams with higher efficiency. Accelerators have now been used in a broad area of science and industry.

The most successful scientific accomplishment in Japan's accelerator-based quantum beam technology in late years is the discovery of nihonium, whose name was formally endorsed by the International Union of Pure and Applied Physics (IUPAP) in November 2016. This is one giant leap for Japan as well as for Asia; nihonium is the first new element discovered by the region. Nihonium (the atomic number, $Z=113$ ) is synthesized by bombarding bismuth $(Z=83)$ atoms with a beam of zinc $(Z=30)$ atoms accelerated in an accelerator. Accelerators used in the field of science had a tendency to be higher and higher in energy so far. This monumental discovery of new element reinforces this tendency. In fact, programs to build gigantic accelerators have now been in progress to generate yet further higher energy.

This tendency to build gigantic accelerators is the exact opposite of industrial applications of accelerators. As illustrated in the advantages of low-energy electron beams in the previous section, industrial applications require the downsizing of equipment. Laser, which belongs to a group of quantum beams, used to be unstable in lasing and show poor lasing efficiency, which made laser devices large-sized. Efforts of improvement aimed at practical application of lasers have enabled emission of reliable and stable laser beams from compact laser devices. Today, essentially there is no obstacle to introducing lasers into production lines or work sites. Think of near-future industrial applications of lasers based on these characteristic properties. Perhaps a technology that we should pay really close attention to as next-generation quantum beams would be a laser acceleration technique proposed by Tajima et al. in 1979. In this technique, radiation of extremely short wavelength and high intensity lasers that exceed $10^{12} \mathrm{~W}$ intensity are focused on an extremely small area to create an area of radiation density exceeding $10^{17} \mathrm{~W} / \mathrm{cm}^{2}$, which gives rise to an extremely high accelerating electric field. The technology is used in this field to accelerate particles such as electrons. Figure 5 outlines this laser acceleration system.

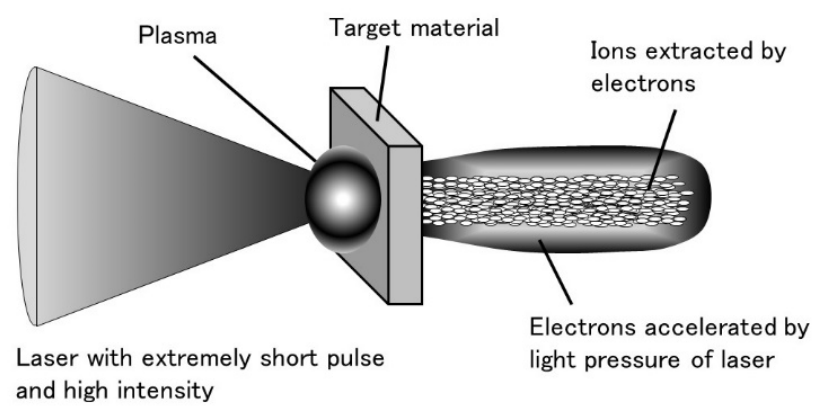

Figure 5. Schematic diagram showing electrons accelerated by pulsed laser irradiation 
Researchers of laser acceleration technologies lately have been busy conducting many trials to create high-energy or more stable lasers typically by creating multiple stages of extremely high accelerating electric fields (Yogo et al., 2017). Laser acceleration is expected to accomplish about one-hundredth size as well as cost of conventional accelerators. This technology if established will allow quantum beams whose quality are similar as those produced by huge accelerators to be available at work sites or production lines at more reasonable cost. It will prompt a broader application of quantum beams than ever before. An example of applications in new areas is remotely controlled nondestructive testing for large structures, such as bridges and piers. Other examples include particle beam cancer treatment equipment, whose beam generation used to rely on huge accelerators. The technology will allow them to be stored in a more compact space, such as underground floors of a building in town.

Further development of laser acceleration, especially those related to extremely short high intensity laser, will ultimately allow realization of dream energy, nuclear fusion (Labaune et al., 2016). Laser acceleration will enable many promising applications. The earliest practical application of laser acceleration is truly desired. We want to keep eye on the development.

\section{Conclusion}

The word "science" has started to be used since around the 17th century to mean credible knowledge that is empirically verified based on experimental results and observations. In contrast, quantum mechanics, which was born toward the end of the 19th century, was knowledge that was hard to observe and verify. Nonetheless, the use of new knowledge of quantum mechanics has contributed to creation of revolutionary products. Examples include semiconductor devices, which have become essential to our life today, X-ray medical examination devices and particle beam devices for cancer treatment.

Meanwhile, this new knowledge created a new area called trans-science (Weinberg, 1972), which refers to a group of questions that can be asked by science but not answered by it. An example of trans-science was nuclear power generation, whose risks and benefits forced us to make difficult political judgment. Times passed while people were struggling to find answers to the group of questions. Then, the Fukushima nuclear disaster occurred. The disaster ended the nuclear "safety myth", and changed many people's views on science and industry.

This trans-science can be applied not only to quantum science but also to the processes of application of advanced technologies, such as artificial intelligence and robots; therefore, we will have to deal with it in real life. These advanced technologies have potential as tools for exploring unknown world, which inevitably contains uncertain elements that nobody knows. As researchers and engineers involved in the research and development of these advanced technologies, we must not leave our future generations at risk to pursue our immediate interest; we must make decisions to build a sustainable society that can continue to flourish. We believe that this is a right thing to do.

In this article, we presented an overview of useful applications and future potential of quantum beams that cannot be accomplished by other technologies, together with the safety issues that should be dealt with in advanced technology application. We hope that this article stimulate interest of many researchers and engineers in quantum beams, and encourage them to get involved in research and development in this field. Ultimately, we do hope that they will help create industries to build a sustainable society in the future.

\section{References}

Fukuda, N. (2017). Characterisics and Applications of Quantum Beam: with Historical Perspective on Utilization of Advanced Technology (in Japanese). Science and industry, 91(9), 274-280.

Furuta, M., \& Fukuda, N. (2016). Current Status and Perspectives Regarding Application of Ionizing Radiation for Sterilization of Food Packaging Materials (in Japanese). Foods \& food ingredients journal of Japan, 221(4), 297-300.

Furuta, M., Suwa, T., Kuwabara, Y., Otsuhata, K., \& Takeda, A. (2002). Electron-beam Sterilization of Laboratory Animal Diets-Sterilization Effect of 10-MeV Electrons from a Linear Accelerator-. Exp. Anim., 51(4), 327-334.

Hoseinimotlagh, S. N., \& Zareie, M. (2014). Calculation of The Deposited Energy and Stopping Range For The Proton, Deuteron and Carbon Beams In Laser Fusion by Fast Ignition. IJFPS, 4(3), 80-88.

Kragh, H. (1999). Quantum generations: A history of physics in the twentieth century. Princeton University Press.

Labaune, C., Baccou, C., Yahia, V., Neuville, C., \& Rafelski, J. (2016). Laser-initiated primary and secondary nuclear reactions in Boron-Nitride. Scientific Reports, 6, 21202. 
Liss, K. D. (2017). Quantum Beam Science-Applications to Probe or Influence Matter and Materials. Quantum Beam Sci., 2017(1), 1. https://doi.org/10.3390/qubs1010001

Tajima, T., \& Dawson, J. M. (1979). Laser Electron Accelerator. Physical Review Letter, 43, 267.

Weinberg, A. M. (1972). Science and Trans-Science. Minerva, 10, 209-222.

Yogo, A., Mima, K., Iwata, N., Tosaki, S., Morace, A., Arikawa, Y., ... \& Sagisaka, A. (2017). Boosting laser-ion acceleration with multi-picosecond pulses. Scientific reports, 7, 42451.

Yu, K. N., Yip, C. W. Y., Nikezic1, D., Ho, J. P. Y., \& Koo, V. S. Y. (2003). Comparison among alpha-particle energy losses in air obtained from data of SRIM, ICRU and experiments. Applied Radiation and Isotopes, 59, 363-366.

\section{Copyrights}

Copyright for this article is retained by the author(s), with first publication rights granted to the journal.

This is an open-access article distributed under the terms and conditions of the Creative Commons Attribution license (http://creativecommons.org/licenses/by/4.0/). 